\title{
Efeitos do Exercício Físico na Qualidade de Vida e Aptidão Física de Pacientes em Hemodiálise
}

\author{
Bruna da Silva Rodrigues, ${ }^{1}$ Juliedy Waldow Kupske, ${ }^{2}$ Renan Felipe Rasia, ${ }^{3}$ \\ Thais Severo Dutra, ${ }^{1}$ Moane Marchesan Krug, ${ }^{3}$ Paulo Ricardo Moreira, ${ }^{1}$ \\ Kalina Durigon Keller, ${ }^{1}$ Rodrigo de Rosso Krug $^{1}$
}

\section{RESUMO}

O objetivo do estudo foi analisar os efeitos do exercício físico sobre a aptidão física e a qualidade de vida de pacientes com insuficiência renal crônica em hemodiálise. Trata-se de um trabalho de intervenção, com uma amostra de 13 pacientes em hemodiálise. O programa foi aplicado em 2019/2 (agosto a dezembro) e ocorria durante as primeiras duas horas da sessão de hemodiálise (intradialítico), três vezes por semana com duração de 20/70 minutos. O mesmo consistia em alongamento ativo, aquecimento, treinamento em cicloergômetro, treinamento contra resistência e alongamento. A aptidão física foi avaliada por meio do teste de seis minutos de caminhada; do teste de sentar e levantar; do teste de flexibilidade, do teste de flexão de cotovelo e da dinamometria. Para avaliar a qualidade de vida foi utilizado o Questionário de Estado de Saúde SF36-V2. Os participantes do programa de exercícios físicos apresentaram melhora em todas as valências físicas avaliadas, ocorrendo de maneira significativa na resistência de membros superiores (pré-teste $=17,85 \pm 4,54$; pós-teste $=20,69 \pm 7,62 ; p=0,034$ ) e na força de preensão manual (pré-teste $=$ $28,15 \pm 9,39$; pós-teste $=28,62 \pm 12,61 ; p=0,048$ ), assim como uma melhora significativa na qualidade de vida nos domínio dor (pré-teste $=73,15 \pm 26,92 ;$ pós-teste $=91,46 \pm 18,78 ; p=0,016$ ), vitalidade (pré-teste $=78,00 \pm 15,48 ;$ pós-teste $=90,08 \pm 9,03 ; p=0,010$ ) e aspectos sociais (pré-teste $=71,31 \pm 28,63$; pós-teste $=85,92 \pm 22,42 ; p=0,050$ ). Concluímos que o programa de exercício resistido traz diversos benefícios para pacientes em hemodiálise.

Palavras-chave: Exercício resistido; aptidão física; reabilitação; força muscular; insuficiência renal crônica; hemodiálise.

\section{EFFECTS OF PHYSICAL EXERCISE ON THE QUALITY OF LIFE AND PHYSICAL FITNESS OF PATIENTS IN HEMODIALYSIS}

\section{ABSTRACT}

The aim of the study was to analyze the effects of physical exercise on physical fitness and quality of life of patients with chronic renal failure undergoing hemodialysis. This is an intervention study, with a sample of 13 patients on hemodialysis at the Renal Clinic of Hospital São Vicente de Paulo. The program was applied in 2019/2 (August to December) and took place during the first two hours of hemodialysis (intradialitic), three times a week lasting 20/70 minutes. The same consisted of active stretching, warm-up, cycle ergometer training, resistance training and stretching. Participants in the physical exercise program showed improvement in all physical valences evaluated, occurring significantly in upper limb resistance (pre-test $=17.85 \pm 4.54 ;$ post-test $=20.69 \pm 7.62 ; p$ $=0.034$ ) and handgrip strength (pre-test $=28.15 \pm 9.39$; post-test $=28.62 \pm 12.61 ; p=0.048$ ), as well as a significant improvement in quality of life in pain domain (pre-test $=73.15 \pm 26.92$; post-test $=91.46 \pm 18.78 ; p=0.016$ ), vitality (pre-test $=78.00 \pm 15.48$; post-test $=90.08 \pm 9.03 ; p=0.010$ ) and social aspects (pre-test $=71.31 \pm 28.63$; post-test $=85.92 \pm 22.42 ; p=0.050$ ). We conclude that the resistance exercise program has several benefits for patients on Hemodialysis.

Keywords: Resistance exercise; physical fitness; rehabilitation; muscle strength; chronic renal failure; hemodialysis.

RECEBIDO EM: 13/1/2021

MODIFICAÇÕES SOLICITADAS EM: 30/4/2021

ACEITO EM: 24/6/2021

\footnotetext{
${ }^{1}$ Universidade de Cruz Alta. Cruz Alta/RS, Brasil.

2 Autora correspondente. Universidade de Cruz Alta - Faculdade de Educação Física de Cruz Alta. Rodovia Municipal Della Campus, Mea Educacional. Cruz Alta/RS, Brasil. CEP 98020-290. http://lattes.cnpq.br/7027998234773332. https://orcid. org/0000-0002-2348-9203.

${ }^{3}$ Universidade Regional do Noroeste do Estado do Rio Grande do Sul - Unijuí. Ijuí/RS, Brasil.
} 


\section{INTRODUÇÃO}

A Insuficiência Renal Crônica (IRC) consiste na diminuição da função renal, diagnosticada pela taxa de filtração glomerular menor que $60 \mathrm{ml} / \mathrm{min}$ por 1,73 $\mathrm{m}^{2}$; albuminúria maior que $30 \mathrm{mg} / 24 \mathrm{~h}$; relação albumina/creatinina maior que $30 \mathrm{mg} / \mathrm{g}$, anormalidades no sedimento urinário, distúrbios eletrolíticos e outras. Estas anormalidades são detectadas por exame histológico e de imagem e devem ser presentes por período maior que três meses. ${ }^{1}$

Os principais fatores de risco para a IRC são a hipertensão arterial sistêmica e a Diabetes Melittus tipo II, seguidos pelas glomerulonefrites. ${ }^{2}$ Segundo as diretrizes da KDIGO, ${ }^{3}$ é importante estabelecer a causa da IRC para decidir o tratamento específico. Existem diferentes tipos de tratamento, entre eles a Hemodiálise (HD), que é o mais utilizado no Brasil; a Diálise Peritoneal, esta pouco adotada, e o transplante renal. ${ }^{2}$

Na análise comparativa dos últimos 10 anos (2009 a 2018) o Censo Brasileiro de Diálise apresenta um aumento no número de pacientes em diálise, de 54,1\% em comparação a 2009, obtendo assim um total de 133.464 pacientes. Do mesmo modo observou-se que a taxa de prevalência e incidência também tiveram acréscimo, com médias de aumento anuais de 23,5 por milhão de população e 6 pmp, obtendo um total respectivo de 640 e 204 pacientes em tratamento por milhão da população. ${ }^{4}$

O tratamento com HD substitui a função fisiológica dos rins, ${ }^{5}$ realizada por uma máquina que filtra o sangue através do dialisador, retirando as substâncias tóxicas, os sais minerais e o excesso de água do organismo. ${ }^{6}$ Pacientes em HD vivenciam um longo tratamento, o que acaba influenciando diretamente em sua rotina, limitando a realização de suas atividades diárias, ${ }^{7}$ ocasionando mudanças no estilo de vida tais como os hábitos alimentares, restrições hídricas e privação do trabalho. ${ }^{8}$

Problemas cognitivos são alguns dos problemas decorrentes da hemodiálise, ${ }^{9}$ redução da capacidade funcional, independentemente do estágio da doença, ${ }^{10}$ processos inflamatórios, acréscimo dos fluidos pulmonares, maior estresse oxidativo, fraqueza muscular, ${ }_{11,12}^{12}$ diminuição da força muscular inspiratória, ${ }^{13}$ baixo nível de atividade física, ${ }_{1}^{14}$ consequentemente apresentam pior aptidão física e baixa qualidade de vida (QV). ${ }^{15}$

A prática de exercícios físicos, tanto aeróbicos quanto resistidos, resultam em efeitos que melhoram a função muscular, a capacidade funcional e também a QV dos indivíduos em HD. Nesta perspectiva, propor programas de exercícios físicos intradialíticos para esta população seria muito importante para a saúde. ${ }^{16}$ Obtendo esse entendimento, a prática de exercícios físicos torna-se um fator de proteção, diminuindo o ritmo de progressão e manutenção renal, acrescido de um avanço no desempenho físico. ${ }^{9}$

Nesse sentido, exercícios físicos realizados durante a HD proporcionam melhora significativa na QV, tanto física quanto emocional. ${ }^{17}$ Outro estudo, ${ }^{18}$ além de confirmar os benefícios citados anteriormente, demonstram uma meIhora no estado geral do paciente em HD, desde um progresso na integridade

Editora Unijuí - Revista Contexto \& Saúde - ISSN 2176-7114 - v. 21, n. 44, out./dez. 2021 
física, psicossocial e bem-estar, reduzindo também a incidência de cãibras, diminuindo complicações em pacientes em HD.

Dessa forma, o objetivo deste estudo foi analisar os efeitos do programa de exercícios físicos na aptidão física e na QV de pacientes com IRC em HD.

\section{MÉTODO}

\section{Tipo de Estudo}

Foi realizado um estudo quali-quantitativo de intervenção. Este estudo faz parte do projeto Probic/Fapergs 2018/2019 (Treinamento Físico para Pacientes em Hemodiálise), Pibex/Unicruz 2018/2020 (Programa de reabilitação física, funcional e cognitiva para doentes renais crônicos em hemodiálise) e é executado pelo Programa de Pós-Graduação em Atenção Integral à Saúde - PPGAIS/Unicruz por meio da dissertação intitulada "Efeitos do treinamento físico na qualidade de vida e aptidão física de pacientes em hemodiálise".

\section{População e Amostra}

A população do estudo foi constituída por todos os pacientes da Clínica Renal do Hospital São Vicente de Paulo/RS, que no ano de 2019 contabilizava um total de 91 pacientes. Já a amostra foi selecionada por conveniência, ou seja, 13 pacientes que aceitaram participar voluntariamente da pesquisa e que tinham tempo de HD superior a três meses.

\section{Programa de Exercícios Físicos}

O programa de exercícios físicos foi realizado durante a HD três vezes na semana, com duração de 20 a 45 minutos e intensidade moderada (escala de Borg entre 6 e 8). O período do treinamento foi de 16 semanas, executado de agosto a novembro de 2019.

O protocolo de atividade é composto por exercícios aeróbicos, resistidos e de alongamento, tanto para membros superiores quanto para membros inferiores, com intensidade moderada (escala de Borg entre 6 e 8). No primeiro momento foi realizada a fase de aquecimento: utilizou-se um aparelho de cicloergômetro por 10 a 15 minutos. No segundo momento foi realizada a fase de fortalecimento, quando foram executados exercícios isométricos e isotônicos com o auxílio de bandagem elástica, halteres e caneleiras. No terceiro momento foi efetivada a fase de relaxamento por meio de práticas de alongamentos passivo e ativo assistido. As atividades foram executadas durante o período intradialítico, nas primeiras duas horas de tratamento, orientadas por alunos bolsistas do programa e supervisionado pelos pesquisadores responsáveis e pelo médico nefrologista da Clínica.

Este protocolo de treinamento está descrito e explicado no estudo de Krug. ${ }^{19}$

Editora Unijuí - Revista Contexto \& Saúde - ISSN 2176-7114 - v. 21, n. 44, out./dez. 2021 


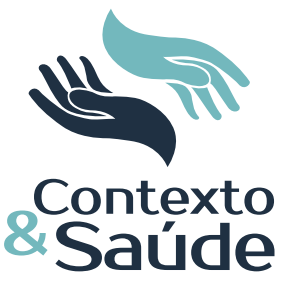

\section{Variáveis e instrumentos}

As variáveis pesquisadas e seus respectivos instrumentos de coleta foram:

- Prontuário físico funcional no qual estavam contidas informações referentes ao tempo de hemodiálise, idade, peso, altura, profissão, doenças associadas, resultados dos testes avaliados e anotações sobre todos os dias de treinamento.

- Teste de seis minutos de caminhada (T6), para avaliar a capacidade funcional. Os pacientes foram instruídos a caminhar o mais rápido possível pelo tempo de seis minutos, verificando a distância percorrida nesse tempo. ${ }^{20}$

- Teste de sentar e levantar (TSL), para avaliar a resistência muscular localizada (RML) de membros inferiores (MI). O paciente permaneceu sentado em uma cadeira com $45 \mathrm{~cm}$ de altura, com as costas eretas, os pés apoiados no solo e afastados na largura dos ombros. Os mesmos levantaram e sentaram durante 30 segundos, sendo registrado o número máximo de repetições. ${ }^{2}$

- Dinamometria para verificar a força de preensão manual: se permaneceu em pé com os dois braços estendidos e antebraço em rotação neutra. A pegada do dinamômetro ajustada individualmente, de acordo com o tamanho das mãos, de forma que a haste mais próxima do corpo do dinamômetro estivesse posicionada nas segundas falanges dos dedos: indicador, médio e anular. $\mathrm{O}$ período de recuperação entre as medidas foi de um cerca de um minuto. 0 teste foi realizado em três tentativas na mão que o participante considerou mais forte. A melhor marca das três tentativas foi utilizada como medida. ${ }^{2}$

- Teste de flexão de braço, para avaliar força e resistência do membro superior, no qual se dá o número de execuções em 30 segundos. Este teste tem como objetivo avaliar a força superior do corpo, necessária para realização de afazeres domésticos e outras atividades que envolvem elevação e transportar coisas como mantimentos, malas e netos. Avaliar a resistência do membro superior: neste teste o participante senta numa cadeira, com as costas eretas, com toda sola dos pés no solo, tronco totalmente encostado. O haltere na mão dominante. $\mathrm{O}$ teste começa com o antebraço perpendicular ao solo. Após tem de fazer o máximo de flexões de antebraço em 30 segundos. Sempre contar flexões totais. Número de rosca bíceps que pode ser concluído em 30 segundos com o peso de 5 libras (2,27 kg) para as mulheres e 8 libras (3,63 $\mathrm{Kg}$ ) para os homens. ${ }^{20}$

- Teste de sentar e alcançar para avaliar a flexibilidade. O teste é realizado com banco de Wells e Dillon, da marca Cardiomed, com precisão de 0,1 cm, que é posicionado no solo e apoiado na parede, permitindo que o sujeito, ao sentar com as pernas estendidas, apoiasse os pés na madeira da caixa. Os avaliados permaneceram sentados, com os pés descalços, apoiados na caixa, sem flexionar os joelhos e deviam inclinar-se para a frente, com as palmas das mãos para baixo e paralelas, até o máximo de sua flexão. A distância máxima alcançada em três tentativas foi registrada em centímetros, como medida de flexibilidade. ${ }^{22}$

- A qualidade de vida foi avaliada pelo Medical Outcomes Study 36 Short Form Health Survey (SF-36), versão validada e traduzida para o Português. ${ }^{23} \mathrm{O}$ instrumento é composto por 36 itens, subdivididos em oito dimensões. Cada dimensão gera um escore que varia de zero a 100 (zero - pior; 100 - melhor).

Editora Unijuí - Revista Contexto \& Saúde - ISSN 2176-7114 - v. 21, n. 44, out./dez. 2021 


\section{Coleta dos Dados}

Primeiramente foi feito o contato com o diretor da clínica solicitando autorização para a realização da pesquisa. Posteriormente os pacientes foram contatados para explicação dos objetivos e convite para participar do estudo. Os pacientes que foram selecionados e aceitaram participar assinaram o Termo de Consentimento Livre e Esclarecido - TCLE. Logo após houve a coleta dos dados pré-intervenção, aplicado o programa de treinamento físico durante 16 semanas e posteriormente foram realizados os pós-testes.

\section{Análise dos Dados}

Os dados quantitativos foram analisados por meio de estatística descritiva, utilizando o programa Stata 11.0, com as variáveis numéricas sendo descritas em virtude de suas médias e desvio padrão, e as variáveis categóricas por meio de porcentagem. O teste " $\mathrm{t}$ " de Student pareado foi utilizado para comparar os valores entre pré e pós-teste. O nível de significância adotado foi de $5 \%$.

\section{Aspectos Éticos}

Este estudo cumpriu todos os princípios éticos de acordo com a Resolução no 466 de 2012, do Conselho Nacional de Saúde, ${ }^{24}$ sendo aprovado pelo Comitê de Ética da Unicruz sob no 4.230.063. Todos os participantes do estudo tiveram de assinar o TCLE e foi garantida a confidencialidade das informações, a participação voluntária e a possibilidade de deixar o estudo a qualquer momento, sem necessidade de justificativa. Os pacientes ficaram com uma cópia do TCLE, e a via assinada foi arquivada na sala do Programa de Pós-Graduação em Atenção Integral à Saúde.

\section{RESULTADOS E DISCUSSÃO}

$\mathrm{Na}$ Tabela 1 fica evidenciado que a média de idade dos pacientes em HD que participaram do programa de exercícios físicos foi de 56,31 $\pm 13,00$ anos, tempo de HD 46,70 $\pm 8,98$ meses. A maioria deles era do sexo feminino e com mais de oito anos de escolaridade.

Tabela 1 - Características sociodemográficas dos pacientes em hemodiálise participantes do programa de exercícios resistidos. Cruz Alta, Rio Grande do Sul, Brasil, 2019 ( $n=13)$

\begin{tabular}{lcc}
\hline Variáveis & Média & Desvio padrão \\
Idade em anos & 56,31 & 13,00 \\
Tempo de hemodiálise em meses & 46,70 & 8,98 \\
\hline & Frequência & Percentual \\
\hline Sexo & & \\
Feminino & 7 & 53,80 \\
Masculino & 6 & 46,20
\end{tabular}


Escolaridade

Fundamental incompleto

Fundamental completo 2

Ensino médio

Ensino Superior

Não respondeu
23,10

15,35

23,10

23,10

15,35

Fonte: Os autores (2019).

$\mathrm{Na}$ Tabela 2 constatou-se que os pacientes em HD ao participarem do programa de exercícios resistidos obtiveram aumento em todas as valências físicas avaliadas. Este aumento, entretanto, ocorreu de maneira significativa na resistência de membros superiores $(p-0,034)$ e na força de preensão manual $(p=0,048)$.

Tabela 2 - Comparação da aptidão física dos pacientes em hemodiálise antes e após a participação no programa de exercícios resistidos. Cruz Alta, Rio Grande do Sul, Brasil, 2019 ( $n=13)$

\begin{tabular}{lccc}
\hline \multicolumn{1}{c}{ Variáveis } & $\begin{array}{c}\text { Pré-teste } \\
\text { Média } \pm \text { DP }\end{array}$ & $\begin{array}{c}\text { Pós-teste } \\
\text { Média } \pm \text { DP }\end{array}$ & p-valor \\
\hline Aptidão cardiorrespiratória & $388,92 \pm 167,98$ & $449,15 \pm 74,49$ & 0,076 \\
Resistência de membros inferiores & $16,00 \pm 5,82$ & $16,77 \pm 2,59$ & 0,127 \\
Resistência de membros superiores & $17,85 \pm 4,54$ & $20,69 \pm 7,62$ & $0,034^{*}$ \\
Força de preensão manual & $28,15 \pm 9,39$ & $28,62 \pm 12,61$ & $0,048^{*}$ \\
Flexibilidade & $26,54 \pm 8,18$ & $27,77 \pm 8,74$ & 0,264 \\
\hline
\end{tabular}

Fonte: Os autores (2019).

Pacientes em HD têm sua força reduzida, o que causa a diminuição da força periférica e limita a capacidade de realização de exercícios físicos, quando comparados com indivíduos saudáveis. ${ }^{25}$ Após as sessões de HD essa redução é ainda mais significante; ao aplicar o teste de dinamometria para verificação da Força de Preensão Manual (FPM) em 156 pacientes de uma clínica em São Paulo, verificou-se uma redução na FPM de 28,6 $\pm 11,4 \mathrm{~kg}$ para 27,7 $\pm 11,7 \mathrm{~kg}$ $(p<0,01) .{ }^{26}$ Esse fato acontece devido à perda de fluidos, além de uremia, anemia, inflamação, processos metabólicos, distúrbios hormonais e nutricionais, bem como alterações na utilização do oxigênio nos tecidos musculares, levando à diminuição da FPM. ${ }^{27,28}$

Um estudo de revisão ${ }^{29}$ mostra que o treinamento de resistência regular com intensidade moderada pode levar à melhora na massa muscular e aumento da força, principalmente para os músculos treinados, o que explica o aumento das valências físicas treinadas. Assim como outra pesquisa sobre benefícios do exercício físico para pacientes em HD conclui que o aumento de força e resistência de membros superiores é um deles, além de ser considerado pelos pacientes de grande importância em sua saúde. ${ }^{30}$

Estudos indicam que pacientes com IRC em HD que têm moderado/alto nível de atividade física possuem maiores níveis de hemoglobina e menores valores de colesterol total. ${ }^{31}$ Além disso, concluíram também que a FPM acima da média era encontrada principalmente em pacientes mais jovens, aqueles que praticavam exercícios físico, possuindo maiores níveis séricos de albumina e creatinina.

Editora Unijuí - Revista Contexto \& Saúde - ISSN 2176-7114 - v. 21, n. 44, out./dez. 2021 
Um estudo realizado por meio de testes do Medical Research Council (MRC), que se baseia na avaliação de membros superiores e inferiores, entre esses a flexão de cotovelo, evidenciou que a força muscular e a capacidade funcional no período pré e pós-exercícios foi verificada uma melhora significativa nos dois aspectos $(p<0,001) .^{32}$

A redução da massa muscular afeta significativamente a força muscular, o que leva a dificuldades nas atividades diárias simples, como as realizadas pelos membros superiores (erguer objetos, por exemplo), juntamente com a força de preensão manual (segurar uma vassoura)..$^{33}$ Este estudo reforça que o fortalecimento muscular atua como prevenção de muitas patologias, uma vez que manter um bom índice de massa muscular e estilo de vida saudável amplia a qualidade de vida, conduzindo a um envelhecimento com mais autonomia.

A prática de exercícios físicos, principalmente os resistidos, causam efeitos positivos no sistema cardiovascular, previne e até reverte a perda da massa muscular, permitindo a eficiência dialítica, melhorando a capacidade funcional, cardíaca, de força e resistência muscular, o que permite um aumento da oxigenação, temperatura, dilatação dos capilares, contração muscular e melhora da circulação, ${ }^{34,35}$ fatores que justificam o presente estudo.

Com o passar do tempo indivíduos treinados adquirem maior capacidade de ativar unidades motoras, assim ativando uma maior quantidade de fibras musculares contráteis sincronizadamente. ${ }^{36}$

$\mathrm{Na}$ Tabela 3 verifica-se que os pacientes ao participarem do programa de exercícios resistidos obtiveram melhora significativa na qualidade de vida nos domínios dor, vitalidade e aspectos sociais.

Tabela 3 - Comparação dos domínios da qualidade de vida dos pacientes em hemodiálise antes e após a participação no programa de exercícios resistidos. Cruz Alta, Rio Grande do Sul, Brasil, 2019 (n=13)

\begin{tabular}{llll}
\hline \multicolumn{1}{c}{ Variáveis } & $\begin{array}{r}\text { Pré-teste } \\
\text { média } \pm \mathrm{DP}\end{array}$ & $\begin{array}{r}\text { Pós-teste } \\
\text { média } \pm \mathrm{DP}\end{array}$ & p-valor \\
\hline Capacidade funcional & $69,77 \pm 27,96$ & $63,46 \pm 20,93$ & 0,242 \\
Limitação por aspectos físicos & $62,23 \pm 34,28$ & $76,77 \pm 16,02$ & 0,064 \\
Dor & $73,15 \pm 26,92$ & $91,46 \pm 18,78$ & $0,016^{*}$ \\
Estado geral de saúde & $61,92 \pm 20,01$ & $66,77 \pm 43,36$ & 0,318 \\
Vitalidade & $78,00 \pm 15,48$ & $90,08 \pm 9,03$ & $0,010^{*}$ \\
Aspectos sociais & $71,31 \pm 28,63$ & $85,92 \pm 22,42$ & $0,050^{*}$ \\
Limitação por aspectos emocionais & $76,77 \pm 16,02$ & $65,23 \pm 40,19$ & 0,465 \\
Saúde mental & $74,85 \pm 27,46$ & $82,31 \pm 29,69$ & 0,180 \\
\hline
\end{tabular}

Fonte: Os autores (2019).

As complicações causadas pela IRC e pelo tratamento em HD afetam os sistemas orgânicos, ocasionando redução dos níveis de força, aumento da gordura corporal e também o perfil lipídico, consequentemente prejudicando a qualidade de vida do paciente..$^{37} \mathrm{Em}$ outra pesquisa ${ }^{38}$ evidenciou-se que o estresse e a ansiedade são fatores que acometem os pacientes de HD. Esse estudo apontou que $71 \%$ dos pacientes encontravam-se estressados e que todos apresentavam ansiedade com níveis moderado (66\%) e severo (34\%).

Editora Unijuí - Revista Contexto \& Saúde - ISSN 2176-7114 - v. 21, n. 44, out./dez. 2021 
A HD acarreta mudanças no estilo de vida, ocasionando limitações físicas, sexuais, psicológicas, familiares e sociais, afetando a qualidade de vida dos pacientes, que em seu cotidiano expressam sentimentos negativos, advindos do prognóstico, da incapacidade, problemas econômicos, da autoimagem, entre outros. Esses pacientes, no entanto, reconhecem que o tratamento possibilita a espera por um transplante renal, dando-lhes uma expectativa de melhora na qualidade de vida. ${ }^{39}$

Ressalta-se que a melhor percepção de QV vem de pacientes em HD que são ativos fisicamente, o que sugere que a prática e bons níveis de atividade física contribuem para essa percepção positiva..$^{40} \mathrm{O}$ protocolo de oito semanas de exercícios para pacientes em HD desenvolvido por Cigarroa et al, ${ }^{41}$ resultou em melhorias na força muscular e na capacidade de caminhada e consequentemente na Qualidade de Vida. Os autores concluem que devido ao progresso na capacidade funcional de pacientes em HD houve uma melhora relativa da QV, pois esta também está associada aos aspectos físicos e à melhora da vitalidade.

Além disso, os pacientes que praticam exercícios físicos relatam que praticar exercícios intradialíticos proporciona melhor convívio durante a HD, além de melhorar a comunicação entre pacientes e equipe médica, tornando o ambiente mais descontraído e agradável. ${ }^{42}$ Isso pode explicar o fato de no presente estudo o domínio aspectos sociais da qualidade de vida tenham aumentado após o treinamento físico.

Praticar exercícios físicos, tanto aeróbios quanto resistidos, leva à ampliação da capacidade funcional, função muscular e qualidade de vida de pacientes de HD, levando ao entendimento de que o treinamento físico deve ser considerado uma modalidade terapêutica importante, fundamentando sua inserção nos centros dialíticos. ${ }^{43} \mathrm{O}$ ganho de massa, força e resistência muscular, aptidão física, qualidade do sono, autopercepção de saúde, capacidade funcional e qualidade de vida são fatores otimizados por meio de programas de exercícios físicos que ocorrem durante a hemodiálise. ${ }^{44}$

Destacam-se algumas limitações do estudo, como o baixo número de participantes e a falta de um grupo controle. Nesse sentido, sugere-se que ensaios clínicos randomizados sejam realizados nesta temática. Como ponto forte do estudo destaca-se os ganhos obtidos pelos pacientes e a exploração de uma nova área de trabalho para o profissional de Educação Física.

\section{CONCLUSÃO}

Conclui-se na presente pesquisa que os pacientes em HD ao frequentarem um programa de exercícios físicos resistidos intradialíticos obtiveram um aumento considerável em todas as valências físicas avaliadas. Esta elevação, porém, ocorreu de maneira significativa na resistência de membros superiores e na força de preensão manual, assim como uma grande melhora na qualidade de vida.

Estes resultados mostram a importância deste tipo de intervenção para auxiliar no tratamento hemodialítico e consequentemente na saúde e qualidade de vida desses pacientes. Além disso, evidencia-se a importância do profissional de Educação Física como membro de uma equipe multiprofissional em saúde em

Editora Unijuí - Revista Contexto \& Saúde - ISSN 2176-7114 - v. 21, n. 44, out./dez. 2021 
clínicas de hemodiálise, tendo em vista que é de total responsabilidade desse profissional a aplicação dos métodos e exercícios para os pacientes, sendo que sob orientação de um profissional da área qualificado, os resultados podem ser maximizados.

\section{REFERÊNCIAS}

${ }^{1}$ Levin A, Stevens PE, Bilous RW, Coresh J, De Francisco ALM, De Jong PE, et al. Kidney disease: Improving global outcomes (KDIGO) CKD work group. KDIGO 2012 clinical practice guideline for the evaluation and management of chronic kidney disease. Kidney Int. 2013;3(1):1-150. doi: https://doi.org/10.1038/kisup.2012.73

2 Thomé FS, Sesso RCC, Lopes AA, Lugon JR, Martins CT. Inquérito brasileiro de diálise crônica 2017. Braz. J. Nephrol. 2019;41(2):208-214.

${ }^{3}$ KDIGO 2012. Clinical Practice Guideline for the Evaluation and Management of Chronic Kidney Disease. Kidney Inter. 2012;3(1)1-163.

${ }^{4}$ Neves PDMM, Sesso RCC, Thomé FS, Lugon JR, Nascimento MM. Censo Brasileiro de Diálise: análise de dados da década 2009-2018. Braz. J. Nephrol. 2020;42(2):191-200.

${ }^{5}$ Silva G, Nóbrega JYL, Nóbrega AL, Araújo RLD, Silva NQ, Nóbrega MF, et al. Percepção de portadores de doença renal crônica com relação ao tratamento hemodialítico. Informativo Semi. [Internet]. 4o de fevereiro de 2015 [citado 29 de set. 2021];9(1):24-30. Disponível em: https://www.gvaa.com.br/revista/index.php/INTESA/article/view/3173

${ }^{6}$ Sociedade Brasileira de Nefrologia (SBN). Hemodiálise. [Acesso em: 22 mar. 2020]. Disponível em: https://www.sbn.org.br/orientacoes-e-tratamentos/tratamentos/hemodialise/

${ }^{7}$ Medeiros RC, Sousa MNA, Santos MLL, Medeiros HRL, Freitas TD, Moraes JC. Perfil epidemiológico de pacientes em tratamento hemodialítico. Rev. Enferm UFPE on-line. 2015;9(11):9846-52.

${ }^{8}$ Araújo JB, Souza Neto VL, Anjos EU, Silva BCO, Rodrigues IDCV, Costa CS. Cotidiano de pacientes renais crônicos submetidos à hemodiálise: expectativas, modificações e relações sociais. J. Res.: fundam. Care. On-line. 2016;8(4):4996-5001.

${ }^{9}$ Roshanravan B, Gamboa J, Wilund K. Exercise and CKD: skeletal muscle dysfunction and practical application of exercise to prevent and treat physical impairments in CKD. Am. J. Kidney Dis. 2017;69(6):837-852.

${ }^{10}$ Oliveira ACF, Vieira DSR, Bündchen DC. Nível de atividade física e capacidade funcional de pacientes com doença renal crônica pré-dialítica e em hemodiálise. Fisioter. Pesqui. 2018;25(3):323-329.

${ }^{11}$ Yılmaz S, Yildirim Y, Yilmaz Z, Kara AV, Taylan M, Demir M, Coskunsel M, et al. Pulmonary Function in Patients with End-Stage Renal Disease: Effects of Hemodialysis and Fluid Overload. Med. Sci. Monit. 2016;22:2779-2784. doi:10.12659/msm.897480

${ }^{12}$ Campos NG, Marizeiro DF, Florêncio ACL, Silva ÍC, Meneses GC, Bezerra GF, Martins $A M C$, Libório $A B$. Effects of respiratory muscle training on endothelium and oxidative stress biomarkers in hemodialysis patients: A randomized clinical trial. Respir Med. 2018;134:103-109. doi: 10.1016/j.rmed.2017.12.005

${ }^{13}$ Zanini SCC, Sperotto MC, Ferreira JS, Piosevan F, Leguisamo CP. Força muscular respiratória e capacidade funcional de pacientes com doença renal crônica em hemodiálise. Fisioter. Bras. 2016;17(5):457-63.

${ }^{14}$ Araújo Filho JC, Amorim CT, Brito ACNL, Oliveira DS, Lemos A, Marinho PEM. Nível de atividade física de pacientes em hemodiálise: um estudo de corte transversal. Fisioter. Pesqui. 2016;23(3):234-40. doi: http://dx.doi.org/10.1590/18092950/14160723032016

${ }^{15}$ Silva MB, Mariot MDM, Riegel F. Qualidade de vida de pacientes renais crônicos em tratamento hemodialítico. Rev. Ciên. Saú. 2020;10(1):11-16. 
${ }^{16}$ Sales CM, Hister FC, Fagundes DS. Atuação fisioterapêutica em pacientes com doença renal crônica. Rev. Cien. F. Ed. M. Amb. 2018;9(2):774-777.

17 Giacomazzi CM, Ritzel C, Birck JA. Fisioterapia intradialítica melhora a qualidade de vida de doentes renais crônicos de um município do sul do país. Rev. Bras. Qual. Vida. 2017;9(4):350-360.

${ }^{18}$ Lisboa LPC, Lima TS, Lopes PS. Abordagem fisioterapêutica em pacientes com insuficiência renal crônica durante a hemodiálise. Rev. Ref. Saúde. 2019;2(3):30-36.

${ }^{19}$ Krug RR, Krug MM, Keller KD, Nicolodi GV, Sturmer G, Sturmer F, et al. Programa de reabilitação físico funcional para pacientes em hemodiálise. Sau. \& Transf. Soc. 2020;11(2):143-150.

${ }^{20}$ Rikli RE, Jones JC. Teste de Aptidão Física para Idosos. Human Kinetics. (Bidutte SRdeC, tradutor), Manole. São Paulo; 2008.

${ }^{21}$ Farias, DL, Teixeira, TG, Tibana, RA, Balsamo, S., Prestes, J. A força de preensão manual é preditora do desempenho da força muscular de membros superiores e inferiores em mulheres sedentárias. Motricidade. 2012;8(2):624-629. Disponível em: https://www. redalyc.org/articulo.oa?id $=273023568074$

22 Wells KF, Dillon EK. O sentar e alcançar - um teste de flexibilidade das costas e das pernas. Pesq. Trimest. 1952;3(1):115-118.

${ }^{23}$ Ciconelli RM; Ferraz MB; Santos W, Meinão I, Quaresma MR. Tradução para a língua portuguesa e validação do questionário genérico de avaliação de qualidade de vida SF36 (Brasil SF-36). Rev. Bras. Reumatol. 1999,39(3):143-50.

${ }^{24}$ Brasil. Conselho Nacional de Saúde. Resolução n 466, de 12 de dezembro de 2012. Aprova normas regulamentadoras de pesquisas envolvendo seres humanos. Brasília: Diário Oficial da União; 2012.

${ }^{25}$ Francisco DS, Brüggemann AKV, Dal Pont T, Lúcio MN, Paulin E. Is the peripheral muscle weakness a limitation to exercise on chronic kidney disease?. Fisioter. Mov. 2020; 33(3355):01-07.

26 Pinto AP, Ramos Cl, Meireles MC, Kamimura MA, Cuppar L. Impacto da sessão de hemodiálise na força de preensão manual. J. Bras. Nefrol. 2015;37(4):451-457.

27 Gomes EP, Reboredo MM, Carvalho EV, Teixeira DR, Carvalho LF, Filho GF. et al. Physical Activity in Hemodialysis Patients Measured by Triaxial Accelerometer. BioMed res. int. 2015;2015:645645. doi: 10.1155/2015/645645

${ }^{28}$ Macdonald JH, Fearn L, Jibani M, Marcora SM. Exertional fatigue in patients with CKD. Am J. Kidney Dis. 2012 Dec.;60(6):930-9. doi: 10.1053/j.ajkd.2012.06.021

${ }^{29}$ Lu Y, Wang Y, Lu Q. Effects of exercise on muscle fitness in dialysis patients: A systematic review and meta-analysis. Am. J. Nephrol. 2019;50(4):291-302.

30 Torres E, Aragoncillo I, Moreno J, Vega A, Abad S, García-Prieto A, Macias N, Hernan$\operatorname{dez}$ A, Godino MT, Luño J. Exercise training during hemodialysis sessions: Physical and biochemical benefits. Ther. Apher. Dial. 2020 Dec.;24(6):648-654. doi: 10.1111/17449987.13469

${ }^{31}$ Kock KS, Neto, JAB, Machado MO. Comparação do nível de atividade física e força de preensão manual com o perfil bioquímico de doentes renais crônicos. Ciência\& Saúde, 2017;10(1):10-17.

${ }^{32}$ Almeida AC, Silva VC, Rezende ARB, Rodrigues ESR, Silveira JM, Miranda EF. Efeitos do protocolo de reabilitação fisioterapêutica na melhora da qualidade de vida e capacidade funcional de pacientes em hemodiálise. Amazônia: Science \& Health. 2016;4(2):9-15.

33 Lima ARS, Portes LA, Oliveira NC, Alfieri FM. Limiar de tolerância de dor à pressão, estilo de vida, força muscular e capacidade funcional em idosas com sarcopenia. Acta Fisiatr. 2016;23(2):73-77.

${ }^{34}$ Freire APCF, Rios CS, Moura RS, Burneiko RCVM, Padulla SAT, Lopes FS. Aplicação de exercício isotônico durante a hemodiálise melhora a eficiência dialítica. Fisioter. Mov. 2013;26(1):167-74. 
35 Souza RMG, Guedes LBA. Benefícios funcionais da fisioterapia para pacientes em hemodiálise. Rev. Pesqui. Fisioter. 2014;4(2):107-113.

${ }^{36}$ Maior AS. Fisiologia dos exercícios resistidos. Phorte Editora LTDA. 2011;1:02-03.

${ }^{37}$ Andrade JC; Araújo SM, Fagundes RM, Felipe TR, Albuquerque Filho, NJB; Pinto EF. Doença Renal Crônica Afeta Negativamente a Composição Corporal, Qualidade de Vida, Perfil Lipídico e a Aptidão Física de Pacientes em Tratamento de Hemodiálise. Motricidade. 2018;14(1):121-133.

${ }^{38}$ Valle LS, Souza VF, Ribeiro AM. Estresse e ansiedade em pacientes renais crônicos submetidos à hemodiálise. Estud. Psicol. (Campinas). 2013;30(1):131-138.

${ }^{39}$ Silva AS, Silveira RS, Fernandes GFM, Lunardi VL, Backes VMS. Percepções e mudanças na qualidade de vida de pacientes submetidos à hemodiálise. Rev. Bras. Enferm. 2011;64(5):839-44. doi: 10.1590/S0034-71672011000500006

${ }^{40}$ Fukushima RLM, Costa JRL, Orlandi FS. Atividade física e a qualidade de vida de pacientes com doença renal crônica em hemodiálise. Fisioter. Pesqui. 2018;25(3):338-344.

${ }^{41}$ Cigarroa I, Barriga R, Michéas C, Zapata-Lamana R, Soto C, Manukian T. Efectos de un programa de ejercicio de fuerza-resistencia muscular en la capacidad funcional, fuerza y calidad de vida de adultos con enfermedad renal crónica en hemodiálisis. Rev. Méd. Chile. 2016;144(7):844-852. Disponível em: ttp://www.scielo.cl/scielo.php?script=sci_arttext\&pid=S0034-98872016000700004\&Ing=es. doi: http://dx.doi.org/10.4067/ S0034-98872016000700004.

${ }^{42}$ Marchesan M, Krug RR, Barbosa AR, Rombaldi AJ. Percepção de pacientes em hemodiálise sobre os benefícios e as modificações no comportamento sedentário após a participação em um programa de exercícios físicos. Rev. Bras. Ciênc. Esporte, 2017;39(3):314-321. Doi: 10.1016/j.rbce.2016.01.012

${ }^{43}$ Nascimento LCA, Coutinho EB, Silva KNG. Efetividade do exercício físico na insuficiência renal crônica. Fisioter. Mov. 2012;25(1):231-239.

${ }^{44}$ Carvalho AR. Sousa IM, Silva DH, Silva AE, Adad RBSF, Silva VO. Os efeitos do exercício físico em pacientes submetidos à hemodiálise: uma revisão sistemática. Rev. Pesq. Fisio [Internet]. 16 mar. 2020 [citado 30 set. 2021];10(2):309-16. Disponível em: https:// www5.bahiana.edu.br/index.php/fisioterapia/article/view/2638 